\title{
Evaluation of LoRa-based Air Pollution Monitoring System
}

\author{
Nael Abd Alfatah Husein ${ }^{1}$, Abdul Hadi Abd Rahman ${ }^{2}$, Dahlila Putri Dahnil ${ }^{3}$ \\ Faculty of Information Science and Technology (FTSM) Universiti Kebangsaan Malaysia (UKM) ${ }^{1}$ \\ Center For Artificial Intelligence Technology, Faculty of Information Science and Technology (FTSM) ${ }^{2}$ \\ Center for Software Technology and Management (SOFTAM) Faculty of Information Science and Technology (FTSM) ${ }^{3}$ \\ Universiti Kebangsaan Malaysia (UKM) ${ }^{2,3}$
}

\begin{abstract}
Air pollution is a threat to human health and the environment. Pollution caused by harmful gases emitted from car exhausts, factories, forest fires and other sources. Carbon monoxide, nitrogen oxides and carbon dioxide are the main elements of air pollution. Serious air pollution may cause harm to our health, thus, a real-time air pollution system to measure existing pollution is needed to classify the pollution level so that appropriate actions can be taken. In a high density area, a big number of sensor nodes are deployed to cover such places and to allow high range communication between nodes and a gateway. This paper presents a real-time and long range air pollution monitoring system for indoor and outdoor environments. The system implemented a wireless sensor network using LoRa technology for data communication between all nodes and sensors. The system consists of three nodes distributed within $900 \mathrm{~m}$ distance to gateway for measuring the concentration of carbon monoxide, carbon dioxide and nitrogen oxide. Experimental results show the system is reliable in both indoor and outdoor applications. The distance coverage achieved up to $900 \mathrm{~m}$ and can be displayed through a web-based system. The experiment with LoRa transmission has shown that the LoRa technology is very suitable for the air pollution system especially in long range transmission compared to other wireless transmission techniques.
\end{abstract}

Keywords-Air pollution; carbon monoxide; wireless sensor network; LoRa; communication; gateway; transmission

\section{INTRODUCTION}

Air pollution has become a threat to the human being as it contains harmful gases from various sources. Exposure to air pollution in open areas accounts for about 3 million deaths per year, and indoor air pollution can cause the same number of deaths. In 2012, indoor and outdoor air pollution was estimated at 6.5 million deaths (11.6\% of the world total). 90 per cent of deaths from air pollution in low- and middleincome countries cause two out of three deaths in South-East Asia and the Western Pacific. Air pollution causes health risks, including cardiovascular disease, stroke, lung cancer and respiratory infections according to world health organization [1]. Malaysia is one of the emerging countries in Southeast Asia facing the challenges of air pollution like other countries in the world. Among the factors that have led to increased air pollution in Malaysia are industrial growth, construction, forest, fires, car fumes and transport, as well as the problem of fog and smoke from the southwest monsoon of forest fires in Indonesia [2]. Air is spread with harmful contaminants such as carbon monoxide, carbon dioxide and nitrogen oxides and is spread both in the open environment and the closed environment. Hence, the importance of the design of the air quality measurement system is flexible and expandable to measure pollutants both in the open or closed environment and provide the user with the value of the pollution existing in one place or several places at the same time.

Air Pollutant Index (API) for health classifications in Malaysia is divided into five categories, each one with a colour and specific value. Classifications are (good, moderate, unhealthy, very unhealthy, and serious) [3]. Traditional air pollution measurement systems lack flexibility and deployment because they are large, stable and require specialized personnel to conduct measurements, in addition to the high cost of creating an air pollution measurement system [4]. With the advent of wireless transmission technologies and sensors, the design of an air pollution measurement system, where sensors and inexpensive Internet of Things (IoT) techniques were used to detect pollution and configuration of wireless networks at remote locations are possible [5].

\section{RELATED WORKS}

Previous studies have been focusing on the integration of the IoT and wireless transmission techniques in monitoring applications. In [6], they implemented a sensor node consists of microcontroller 18F4550 with sensors $\mathrm{CO} 2, \mathrm{O} 2, \mathrm{SO} 2-\mathrm{D} 4$, $\mathrm{CO}, \mathrm{NO} 2$, temperature and humidity. The data were sent to the gateway by Zigbee modules. The disadvantages of this system are that the use of Zigbee technology as a mean of transmitting data between the sensor node and gateway. Zigbee technology is limited to about 100 meters' transmission and consumes high energy. In [7], the study has introduced a mobile node designed to be placed in a vehicle and the data of the pollution sensor were sent in real-time. The use of this method is very expensive in addition to the continuous payments due to its dependence on the mobile network; furthermore, the weakness of the cellular network in the traffic areas and the absence of network coverage in rural areas.

The wireless network provides an alternative solution where it consists of nodes distributed in different places in a closed environment. These nodes transmit pollution data depending on the sensors attached to each node to the base station [8], Moreover, these nodes use low power controllers. However, this system is costly and required all nodes to be 
working to provide the total measurement in the enclosed environment. Another solution is using GPRS-based system as in [9], it was designed to measure polluting gases: carbon monoxide, carbon dioxide and sulphur dioxide. Data are displayed in the Google Maps interface, the study has used to the 16-bit microcontroller and air pollution sensors: carbon dioxide, NO2, sulphur dioxide, GPS module and GPRS modem. The disadvantage of this system is that the measuring air pollution process takes place in an open area and relying on cellular network technology. This reliance has many disadvantages like the limited coverage of the cellular network, the weak mobile network in rural or remote areas, and high cost.

With the advances of network technology in wireless sensor networks (WSN), air quality measurement systems would rather be designed to send data to the main gateway or base node to collect sensors data. It WSNs consists of several sensor nodes scattered in large areas based on wireless communication. The function of the sensor node is to measure physical phenomena such as temperature and humidity with limited memory [10]. The sensor node, in terms of physical components, consists of a wireless transmitter/receiver, an embedded processor, internal and external memory, a power source and sensors [11]. There are many algorithms that regulate wireless network operations in terms of sending data from different nodes [12]. Sensor node has the ability to process data and storage. It contains sensors and each node has the ability to generate, analyse and direct data to a certain point. Wireless sensor networks are used in many applications like agricultural, industrial, medical and others [13]. WSN collects information from different sensors in large and small networks so that end users can access and process data. These networks can be used to monitor people's health, weather conditions, traffic control and air pollution [14]. WSN consists of the base station, where these sensor nodes sense data from the environment and direct data back to the base station either through a single hop or multiple hop technology that is sent to the user via other secondary links [15].

LoRa is a long-range wireless communication protocol transfer rates range between $290 \mathrm{bps}$ and $50 \mathrm{kbps}$. LoRa peripherals are used to communicate with gateways. LPWAN (Wide-Area Low Power Area Network) technologies are increasingly on-demand [16]. LoRa is a low-power wireless network protocol (LPWAN) in a wide area for Internet Objects (IoT) applications. The effective, long-term, cost and power of LPWANs make this technology the most suitable for smart city applications [17]. Low-Power Area Networks (LPWANs) are the types of networks that are used to connect Internet objects from a wide range of sectors. These types of technologies provide devices internet objects (IoT) the ability to transfer a small number of data to long ranges, taking into consideration the minimum power consumption [18]. Some $2.4 \mathrm{GHz}$ wireless technologies such as Bluetooth, Wi-Fi and Zigbee consume a lot of power and only support the transmission range between the nodes and the gateway for up to 100 meters [19]. Therefore, if nodes are to be deployed to detect pollution in a large area, using a $2.4 \mathrm{GHz}$ technology, the node battery will not be enough to be used without recharging continuously. The deployment of a wireless sensor network based on this technology will be expensive and ineffective. In addition, when using these nodes within a wireless network spread over wide distances, the biggest problem we face is the limited distance of communication because if we want a distance greater than 100 meters, it will require frequent gates to re-send or receive data to increase the coverage area. This means that increasing the number of gates to cover a larger distance will result in a direct increase in the cost of the project.

This paper focuses on the configuration of a wireless network that contains two important aspects: energy and cost. The system is distinguished by low power, long coverage and long battery life due to our reliance on LoRa transmission technology. We propose a system for data observation and air pollution analysis in indoor and outdoor environments. Pollution measurement data can be collected from different locations by connecting to a special site. We use LPWAN and LoRa for this purpose and we rely on Malaysia API classification in the classification of measured pollution.

\section{PROPOSED SYSTEM}

This section shows the structure of the system and the explanation of the physical components used in the project. The nodes in this system are responsible for detecting pollution and used three sensors to measure carbon monoxide, carbon dioxide and nitrogen oxides. The nodes are deployed in different areas; thus, they provide enough information on the percentage of pollution and classify them based on API Malaysia. The node sends data to the gateway. The data collected by the gateway are analysed and presented to users in an easy-to-use platform. A sensor node is made from the Arduino UNO R3 microcontroller board. This is the main part of the system, where all the codes used to regulate the work and the functions of the components attached will be stored. The advantages of the system presented in this study are low cost, easy to use and microcontroller based on ATmega328. For the sensors, the study used three sensors (MQ7-MQ2MQ135), sensors have been selected as they can detect major gases in case of pollution: carbon monoxide, carbon dioxide and nitrogen oxides. The range of detection of gas sensors ranges from $10 \mathrm{ppm}$ to $1000 \mathrm{ppm}$ and from $10 \mathrm{ppm}$ to 300 ppm, respectively.

The transmitter module used in the study is RFM LoRa Shield, it is compatible with Arduino. It allows users to send data and access to extremely long ranges at low data rates ( $<50 \mathrm{kbps}$ ). LoRa shields Provide long-range connectivity with diffusion spectrum and high immunity to interfering with current consumption reduction [20]. In the power module, the study used $1200 \mathrm{~mA}$ 3.7-volt rechargeable Li-ion battery. For the second part of the system, the gateway is responsible for collect data of the nodes; we used RFM LoRa Shield with Arduino as is shown in Fig. 1.

To visualize the data, we created a web page to display the pollution information. The information was displayed in a local server where they can be available on-site and mobilerelated applications as shown in Fig. 2. The system displays the rates of carbon monoxide, carbon dioxide and nitrogen oxides pollution with a hazard rating according to the global standard in Malaysia. Also, they will be aware of the 
proportions of those gases in the air. This system provides accurate information, which has been tested in the indoor and outdoor environment, to identify the quality of gases in the air that causes pollution and thus can take actions to reduce those sources. The system will provide information at the lowest cost. The user interface is simple to use and understandable. The system will update the data every minute from different locations.
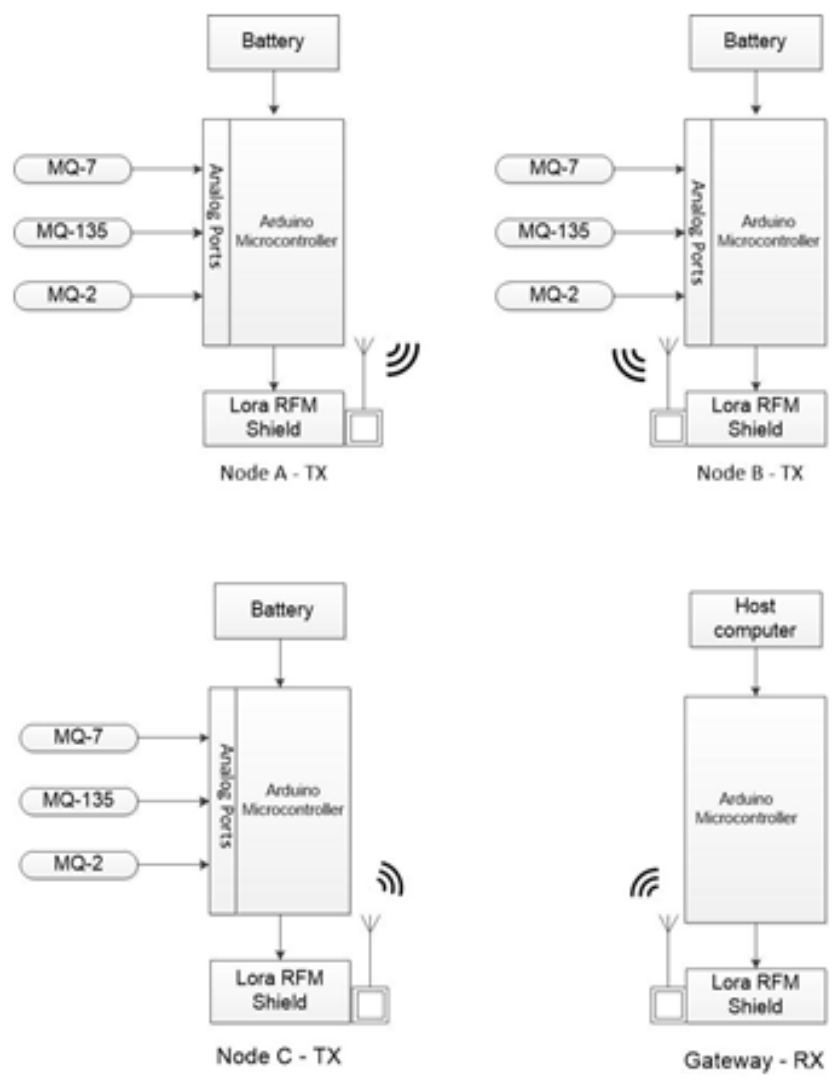

Fig. 1. Air Pollution Monitoring System.

(i) localhost/nail/index2.php

\section{System Air Quality}

Pollution measurement for three zones $(\mathrm{A}, \mathrm{B}, \mathrm{C})$

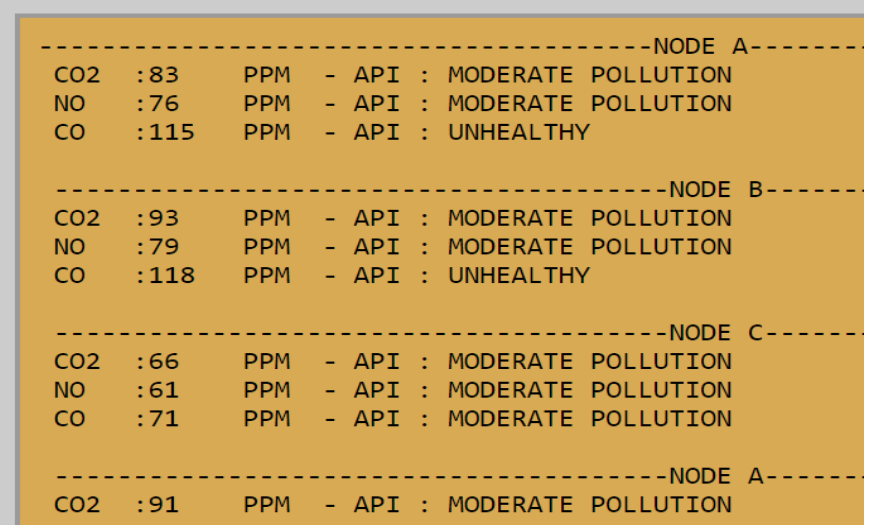

Fig. 2. Web-Based user Interface.
This system is capable of detecting and classifying pollution, by establishing a wireless sensor network to provide information, requires avoiding the disadvantages of previous measurement systems in terms of using wireless communication technologies between network components. Traditionally, this type of network can be configured, but multiple gateways are needed because of limited of wireless transmission. All nodes were deployed at different distances to measure pollution in real-time. Data is obtained every one minute from the nodes that are published and collected by the gateway. The collected data were stored in a text file which represents the database on the hard drive and then transfers it to end-users through a local server.

\section{RESUlTS AND DISCUSSION}

In this study, four experiments are conducted: (A) validation of the proposed system to measure pollution by sensors and the ability of the wireless network to send and receive data between the nodes and the gateway, (B) pollution measurements in an outdoor environment, (C) pollution measurements in indoor environment and (D) maximum distance coverage for LoRa data transmission between the nodes and the gateway.

\section{A. Pollution Classification}

The system was first tested where the sensors connected to node A were exposed to some pollutants gradually, and the data was sent wirelessly to the gateway. Fig. 3 shows the systematic classification of the pollution measured by the sensors according to the degree of measured pollution. The results show the ability of the system to record the level of pollutions.

\section{B. Outdoor Environment}

Two experiments were conducted to test the system in the outdoor environment. The first experiment is in University Kebangsaan Malaysia (UKM) Campus and in Kajang city centre.

1) A. UKM campus experiment: In this experiment, three nodes were deployed in different areas to measure pollution. Node A was placed at a crossroads opposite the main gate of UKM University, node B was placed on the main road in the campus and node $\mathrm{C}$ was placed at the building Dewan Canselori Tun Abdul Razak (DECTAR) as shown in Fig. 4. Fig. 5 illustrates the distance between all three nodes and the gateway at UKM University.

The results collected by gateway showed that node A, which was located at a crossroads outside UKM University, has the highest in pollution measurement compared to the other two nodes where the pollution with carbon dioxide is 43 ppm, carbon monoxide and nitrogen oxides are $19 \mathrm{ppm}$. The pollution was classified as "good low pollution". Pollution within the university area measured in B and $\mathrm{C}$ is classified as "good low pollution", the lowest level of pollution was in node $\mathrm{C}(\mathrm{CO} 2=4 \mathrm{ppm}, \mathrm{NO}=12 \mathrm{ppm}, \mathrm{CO}=11 \mathrm{ppm})$ as the building is far from the main road which contributes to low pollution rates. Fig. 6 presents pollution measurement results from three nodes. 


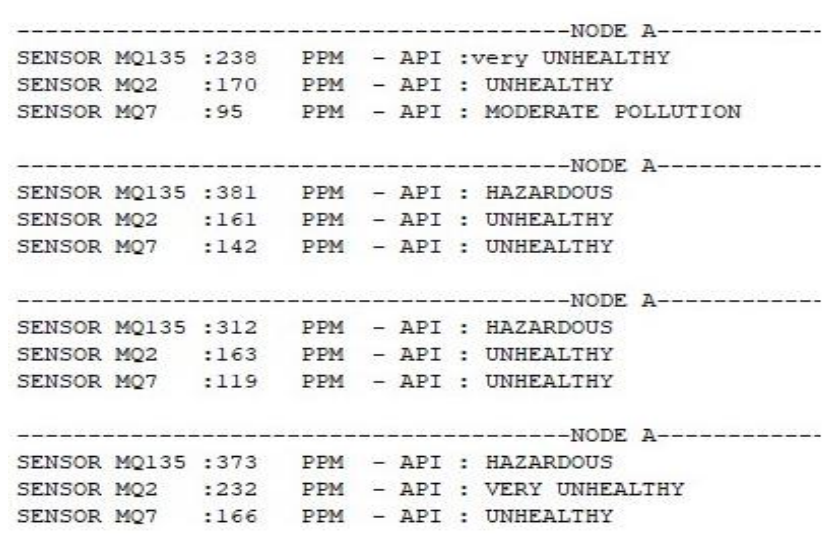

Fig. 3. Air Pollution Measurement and Classification.

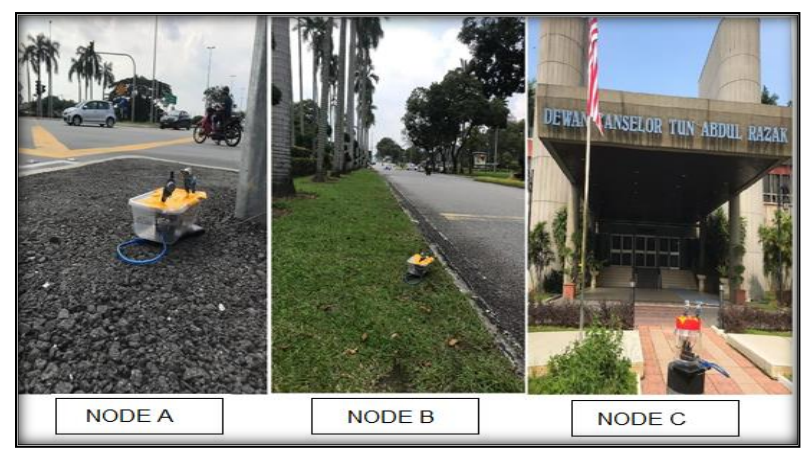

Fig. 4. Placement of Nodes in UKM Campus.

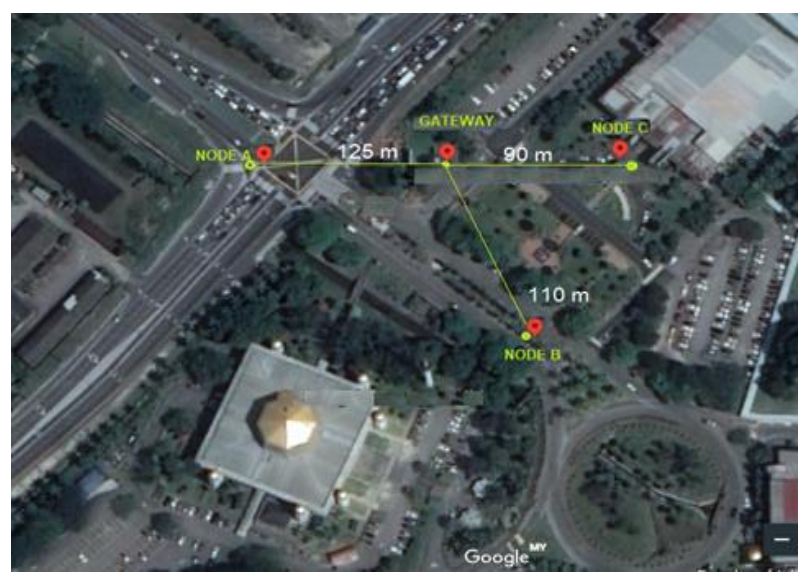

Fig. 5. Distance between Nodes and Gateway in UKM University.

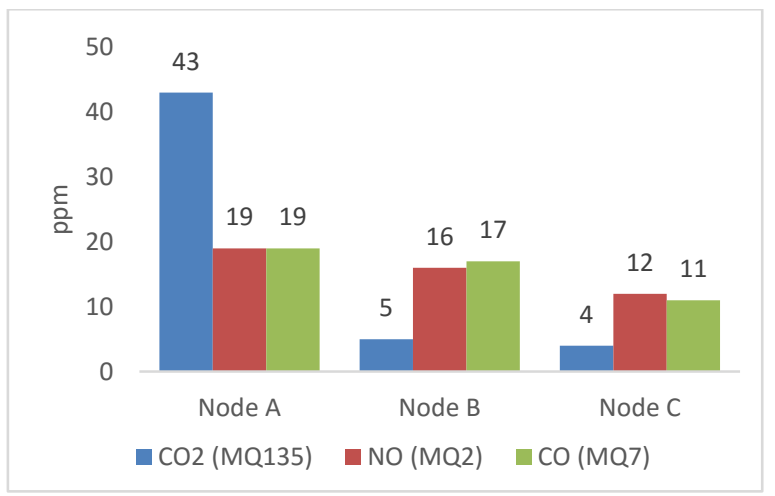

Fig. 6. Pollution Sensor Reading in UKM Campus.

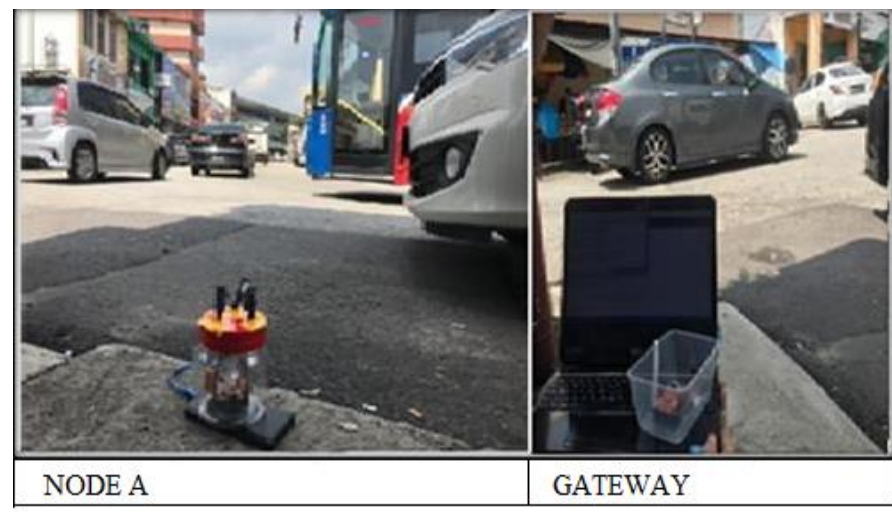

Fig. 7. Node A and Gateway Location in Kajang City Centre.

2) B. Kajang city center: An experiment was conducted to measure pollution in Kajang area that has many people, with a variety of activities and with more vehicles on the road. All sensors attached to the nodes provide information on the gases reading in this area. Fig. 7 shows the placement of nodes at the main street near the Kajang MRT station (SBK34). The recorded pollution data are shown in Fig. 8. It is shown that the pollution of carbon dioxide was $82 \mathrm{ppm}$ while the pollution with nitrogen oxides was $65 \mathrm{ppm}$ and carbon monoxide was $52 \mathrm{ppm}$, thus, the pollution level was classified as moderate. A higher pollution level is recorded as the node is next to the main road where traffic congestion is high most of the time.

\section{Indoor Experiment}

Another testing was performed in an indoor environment with less air circulation, such as offices, classrooms and libraries that use central air conditioning where windows and doors are closed. The sources of pollution come from carbon dioxide associated exhaled while breathing, central air, or the use of air fresheners. Pollution measurements were placed in the faculty library as illustrates in Fig. 9, which contained halls of different sizes. Two halls were chosen for the experiments, a hall with $(3 \times 6 \mathrm{~m})$ size with the presence of three students and the other one hall with a $(5 \times 15 \mathrm{~m})$ size with a presence of four students, the library uses central air conditioners where windows and doors inside the halls are closed.

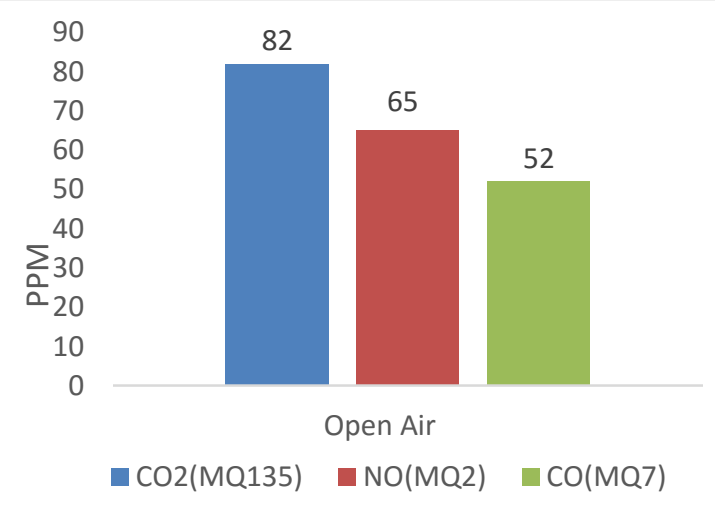

Fig. 8. Pollution Sensor Reading in Kajang City Centre. 


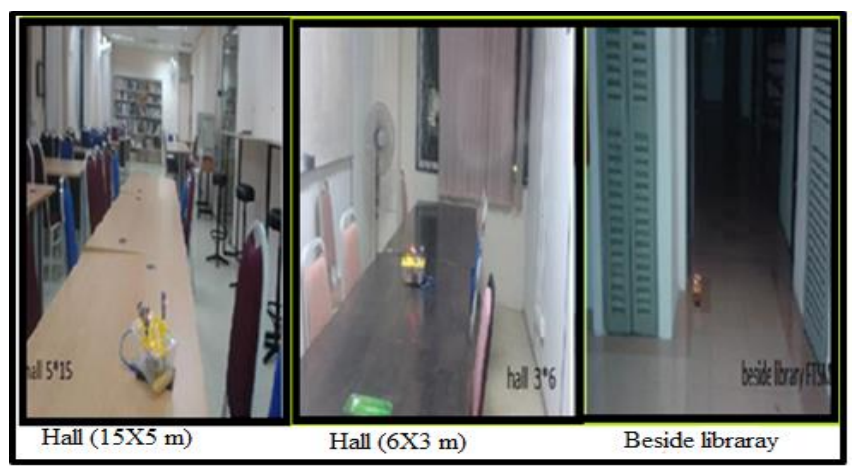

Fig. 9. FTSM Library Halls Selected for the Experiment.

3) A. Hall $(5 \times 15 \mathrm{~m})$ : Node A was placed outside a library to measure outdoor pollution whereas node $\mathrm{B}$ was placed in a 75-meter closed hall with 3 students while node $C$ was placed in a hall of the same size but without students. The gateway was placed inside the library to receive sensor data from the three nodes.

The results showed that during the presence of three students in the hall, the carbon dioxide ratio was $32 \mathrm{ppm}$ with a slight increase in the ratio of carbon monoxide and nitrogen oxides. This was classified as a low pollution level and is classified as good. Carbon dioxide was detected in the closed due to student respiration, while the ratios of carbon monoxide and nitrogen oxides were equal to those in the air. Fig. 10 shows the proportions of pollution measured by nodes.

4) B. Hall $(6 \times 3 \mathrm{~m})$ : Node A was placed outside the library while node $\mathrm{B}$ was placed in a closed hall of $18 \mathrm{~m}$ with 3 students while node $\mathrm{C}$ was placed in a hall of the same size but without students. The gateway was placed inside the library to receive sensors data from the three nodes. The results showed that the pollution of nitrogen oxides and carbon monoxide in the library was caused by internal indoor pollution. The nitrogen oxides and carbon monoxide inside the library were equal to those found in open-air measured by node A. The outdoor carbon dioxide ratio was $10 \mathrm{ppm}$. In the hall with the three students, a $47 \mathrm{ppm}$ ratio was measured by node B due to breathing. The room was empty and the reading rose to $47 \mathrm{ppm}$ in the hall with the three students because of the breath measured by node B. In another situation where the hall was empty, the ratio of carbon dioxide decreased to 27 ppm compared with student's hall and became $20 \mathrm{ppm}$ as shown in Fig. 11.

\section{LoRa Data Transmission}

An experiment was conducted at the UKM Stadium to assess the scope of the LoRa transmission coverage. The experiment was conducted at a different football field where the ground was flat and contained trees and buildings. The temperature was $36^{\circ} \mathrm{C}$, the humidity was $86 \%$. The gateway was placed at the end of the field and the knot started from a distance of zero to $1.1 \mathrm{~km}$. The number of packets received from the node is counted for 2 minutes at 100 meters. The measurement was conducted in 12 locations (from 0 to 1100 $\mathrm{m})$ as shown in Fig. 12. The experiment showed that the reception rate was $100 \%$ at a distance of 0 to $900 \mathrm{~m}$. There were no missing packets. After that distance $(900 \mathrm{~m})$, there was a loss rate of $95 \%$ at $1 \mathrm{~km}$ and $1.1 \mathrm{~km}$. The loss of the number of packets increases after $900 \mathrm{~m}$ as shown in Fig. 13.

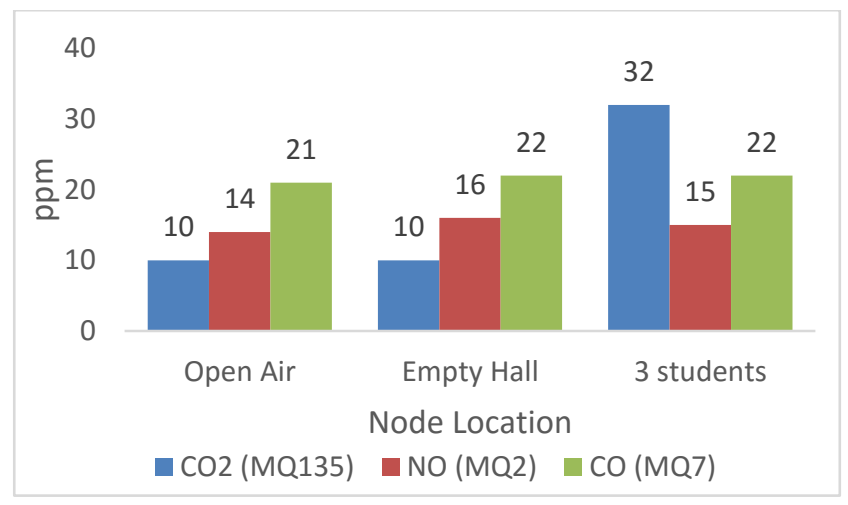

Fig. 10. Air Pollution in the Hall (5x 15m).

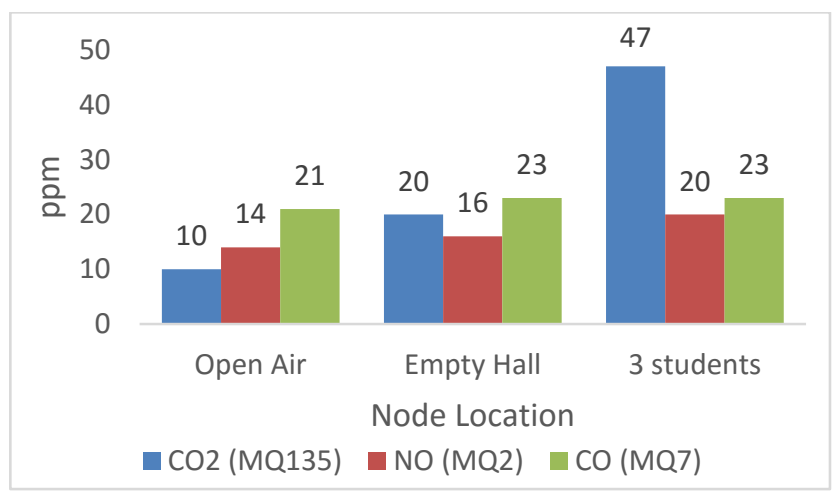

Fig. 11. Air Pollution in the Hall (6X3m).

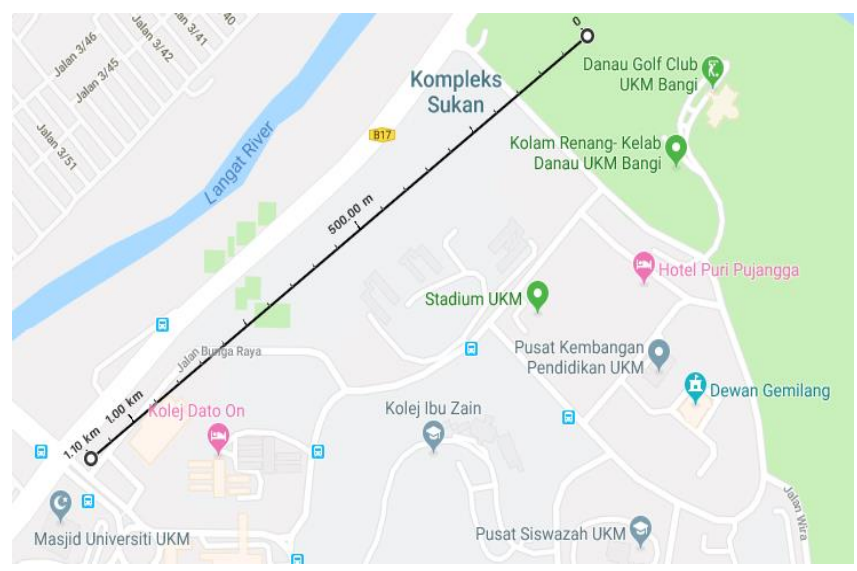

Fig. 12. Route of the LoRa Performance Test. 


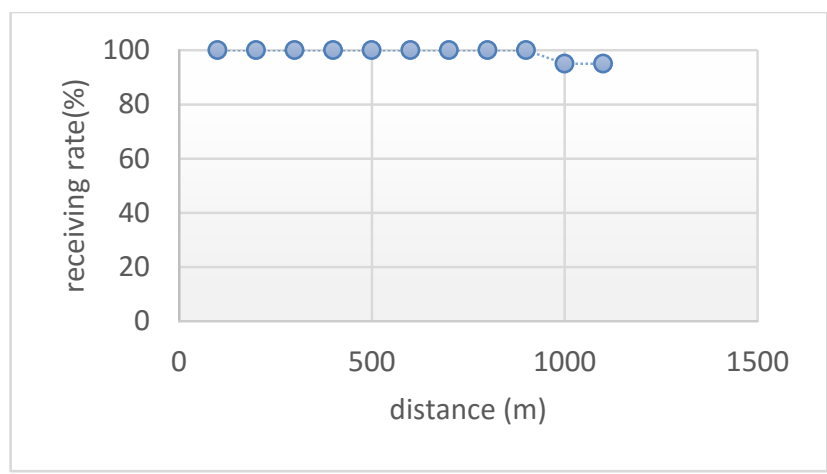

Fig. 13. LoRa Receiving Rate between Gateway and Node.

\section{CONCLUSION AND FUTURE WORKS}

The proposed environmental pollution monitoring system has overcome many of the disadvantages of traditional pollution measurement systems, which also rely on wireless transmission technologies that are no more than 100 meters' coverage, such as Zigbee and Wi-Fi. The proposed system is a low-cost system, does not require any phone/data bills as for cellular-based system, small size, long coverage and transmitting in real-time. Based on the practical experiments in the indoor and outdoor environment, the system has proven its ability to detect pollution and classify its pollution level. The experiment with LoRa transmission has shown that LoRa technology is very suitable for the air pollution system especially in long-range transmission compared to other wireless transmission techniques.

Future studies can focus on enhancing the coverage of LoRa technology by adding more gateways in trafficcongested areas. In addition, new types sensors can be added to detect other relevant types of gases.

\section{ACKNOWLEDGMENT}

The research is funded by Universiti Kebangsaan Malaysia (UKM) research project GUP-2017-068 and DCP-2018-001/2 under Research Centre for Software Technology and Management (SOFTAM), www.ftsm.ukm.my/softam, Faculty of Information Science and Technology.

\section{REFERENCES}

[1] Organization, W. H. 2018. WHO releases country estimates on air pollution exposure and health impact. World Health Organization: Geneva, Switzerland. https://www.who.int/news-room/detail/27-092016-who-releases-country-estimates-on-air-pollution-exposure-andhealth-impact [5 September 2018].

[2] Awang, M. Bin, Jaafar, A. B., Abdullah, A. M., Ismail, M. Bin, Hassan, M. N., Abdullah, R., Johan, S., et al. 2000. Air quality in Malaysia: impacts, management issues and future challenges. Respirology 5(2): 183-196.
[3] Aziz, R. M., Zabawi, A. G. M., Azdawiyah, A. T. S. \& Fazlyzan, A. 2018. Effects of haze on net photosynthetic rate, stomatal conductance and yield of Malaysian rice (Oryza sativa L.) varieties. J. Trop. Agric. and Fd. Sc 46(2): 157-169.

[4] Ya'Acob, N., Azize, A., Adnan, N. M., Yusof, A. L. \& Sarnin, S. S. 2016. Haze monitoring based on air pollution index (API) and geographic information system (GIS). Systems, Process and Control (ICSPC), 2016 IEEE Conference on, hlm. 7-11. IEEE.

[5] Kumar, S. \& Jasuja, A. 2017. Air quality monitoring system based on IoT using Raspberry Pi. Computing, Communication and Automation (ICCCA), 2017 International Conference on 1341-1346.

[6] Kumar, A., Hancke, G. P. \& Member, S. 2014. Low Cost Requirements. IEEE Sensors Journal 14(8): 2557-2566.

[7] Devarakonda, S., Sevusu, P., Liu, H., Liu, R., Iftode, L. \& Nath, B. 2013. Real-time air quality monitoring through mobile sensing in metropolitan areas. Proceedings of the 2nd ACM SIGKDD international workshop on urban computing, 15. ACM.

[8] Preethichandra, D. M. G. 2013. Design of a smart indoor air quality monitoring wireless sensor network for assisted living. 2013 IEEE International Instrumentation and Measurement Technology Conference (I2MTC). 1306-1310. IEEE.

[9] Al-Ali, A. R., Zualkernan, I. \& Aloul, F. 2010. A mobile GPRS-sensors array for air pollution monitoring. IEEE Sensors Journal 10(10): 16661671.

[10] Gangurde, P. \& Bhende, M. 2015. A review on precision agriculture using wireless sensor networks. International Journal of Engineering Trends and Technology 23: 426-431.

[11] Wang, Q. \& Balasingham, I. 2010. Wireless Sensor Networks - An Introduction. doi:10.5772/13225.

[12] Habib, M. A., Arafat, M. Y. \& Moh, S. 2018. A Survey on Reinforcement-Learning-Based Routing Protocols in Wireless Sensor Networks.

[13] Sohraby, K., Minoli, D. \& Znati, T. 2007. Wireless sensor networks: technology, protocols, and applications. John Wiley \& Sons.

[14] Kiani, F. \& Seyyedabbasi, A. 2018. Wireless sensor network and internet of things in precision agriculture. Int. J. Adv. Comput. Sci. Appl 9(8): 220-226.

[15] Rani, A. \& Kumar, S. 2017. A survey of security in wireless sensor networks. 2017 3rd International Conference on Computational Intelligence \& Communication Technology (CICT), hlm. 1-5. IEEE.

[16] LoRaWAN-A low power WAN protocol for Internet of Things: A review and opportunities.

[17] Noreen, U., Bounceur, A. \& Clavier, L. 2017. A study of LoRa low power and wide area network technology. 2017 International Conference on Advanced Technologies for Signal and Image Processing (ATSIP),. 1-6. IEEE.

[18] Ayoub, W., Samhat, A. E., Nouvel, F., Mroue, M. \& Prévotet, J.-C. 2018. Internet of Mobile Things: Overview of LoRaWAN, DASH7, and NB-IoT in LPWANs standards and Supported Mobility. IEEE Communications Surveys \& Tutorials.

[19] Pothuganti, K. \& Chitneni, A. 2014. A comparative study of wireless protocols: Bluetooth, UWB, ZigBee, and Wi-Fi. Advance in Electronic and Electric Engineering 4(6): 655-662.

[20] Lavric, A. \& Popa, V. 2017. Internet of things and $\mathrm{LoRa}^{\mathrm{TM}}$ low-power wide-area networks: a survey. Signals, Circuits and Systems (ISSCS), 2017 International Symposium on, hlm. 1-5. IEEE. 